\title{
A VEGETAÇĀO DA MATA RIBEIRINHA NO CURSO MÉDIO DO RIO JACUI, RS
}

Solon Jonas Longhi, Miguel Antão Durlo e José Newton Cardoso Marchiori Departamento de Ciências Florestais. Centro de Ciências Rurais.UFSM. Santa Maria, RS.

RESUMO

A composição florística e estrutura da mata ribeirinha na região do curso médio do Rio Jacuí foi caracterizada pela anālise de 10 amostras. Encontrou-se uma alta heterogeneidade, grande densida de de indivíduos e presença de algumas espécies exclusivas para es te tipo florestal na região.

\section{SUMMARY}

LONGHI,S.J.; DURLO,M.A. and MARCHIORI,J.N.C., 1982. The river bank vegetation in the mid flow of the Jacuí River, RS. Ciencia e Natura (4):151-161.

The floristic composition and wood structure of the river bank of the Jacuí River mid flow was characterized through the analysis of ten samples. A high heterogene, large individual density and presence of some exclusive species for this type in the region, were found.

INTRODUÇÃOO

0 vale do curso médio do Rio Jacuí, originalmente, estava coberto.por uma densa mata subtropical. A ocupação agrícola, nesta região, provocou um intenso desmatamento, restando atualmente uma cobertura florestal bastante reduzida. As āreas ainda com vegetação florestal localizam-se, principalmente, em encostas de difícil aces so, em faixas descontínuas às margens do Rio Jacuí, ou em pequenas ilhas.

Como a proximidade do rio fornece condições especiais para o desenvolvimento de certas espēcies, procurou-se estudar separada mente este tipo florestal, que foi denominado de Mata Ribeirinha.

\section{REVISAO DE LITERATURA}

A caracterização de um tipo florestal deve ser fundamenta da, segundo RIZZINI (14), em caracteres fisionōmicos e ecológicos.

A vegetação natural ocorrente às margens dos rios apresen ta, devido à condições ecolōgicas específicas, uma composição florís tica e uma fisionomia singular, suficiente para caracterizá-la como um tipo florestal prōprio. 
E por este motivo, que na literatura fitogeogräfica encon tram-se termos como Mata de Igapó (HUECK, 9), Floresta Ripāria, Mą ta de Galeria e Mata Ciliar (RIZZINI, 15) para denominar a vegetação associada aos rios. O primeiro destes termos ē de uso restrito para a Mata Tropical Pluvial da Amazōnia e os restantes, para a vegetação florestal associada aos rios em regiões campestres. Em virtude dis so, preferiu-se denominar a mata em estudo de Mata Ribeirinha, por se tratar de uma vegetação que não é bem definida pela terminologia usua 1 .

Os mētodos de anālise estrutural de Vegetação baseiam-se, normalmente, em cálculos de Abundāncia, Dominância e Frequência, con forme recomenda CAINE \& CASTRO (3). BRAUN-BLANQUET (1) aconselha 0 uso do Valor de Cobertura (Abundância + Dominância Relativas), ten do em vista que a Frequência tem uma influência na hierarquia das es pēcies constituintes de um povoamento, quando estas se encontramuni formemente distribuĩdas.

A Abundância, segundo LAMPRECHT $(10,11)$, è o número de ín dividuos das diferentes espécies existentes na floresta, referido por unidade de ārea (geralmente em hectare). A Abundāncia Relativa indi ca a participação das espēcies em percentagem do nūmero total de ār vores levantadas.

Segundo FONT-QUER (7) e FORSTER (8), Dominância è a medi da de projeção total do corpo das ārvores. Como em florestas muito densas ē difícii e praticamente impossível determinar os valores de projeção horizontal das copas das ārvores, CAINE \& CASTRO (3) suge rem que se utilize a Ârea Basal dos fustes como substituição à ārea de projeção das copas, jā que existe estreita correlação entre ambas. Tal correlação foi encontrada por BRÜIG \& HEUVELDOP (2), LONGHI(12)e VOLKART (16) entre outros.

A regeneração natural è tambēm um critērio importante para a caracterização da vegetação e compreensão de sua dinâmica, como con sideram FINOL $(4,5,6)$ e PETIT (13).

\section{MATERIAL E METODOS}

Esta pesquisa foi desenvolvida na ārea a ser inundada pela Barragem de Dona Francisca, às margens do Rio Jacuí e Jacuizinho, Estado do Rio Grande do Sul.

0 levantamento da vegetação foi realizado em 10 amostras retangulares de $200 \mathrm{~m}^{2}(10 \times 20 \mathrm{~m})$, dispostas paralelamente ao le to do rio e distribuídas ao acaso na ārea de estudo.

A regeneração natural foi avaliada por levantamentos em sub-amostras quadrangulares com ārea de $9 \mathrm{~m}^{2}$. 
RESULTADOS E DISCUSSÃO

A mata ribeirinha apresenta um nümero elevado de espécies lenhosas (Tabela I). Este fato deve-se a ocorrência de vārias espé cies adaptadas a condições ecológicas particulares do local, além das entidades botânicas menos exigentes e de maior plasticidade.

TABELA I. NOME CIENTIFICO E FAMTLIA BOTÃNICA DAS ESPECIES LENHOSAS.

\begin{tabular}{|c|c|c|}
\hline Nọ & NOME CIENTIFICO & FAMILIA \\
\hline 1 & Acacia recurva Benth. & Leguminosae \\
\hline 2 & Actinostemom concolor (Spreng.) Md11. Arg. & Euphorbiaceae \\
\hline 3 & Alchornea triplinervia(Spreng.) Mul1. Arg. & Euphorbiaceae \\
\hline 4 & AlZophylus edulis (Camb.) Radlk. & Sapindaceae \\
\hline 5 & Apuleia Zeiocarpa (Vog.) Macbr. & Leguminosae \\
\hline 6 & Ateleia glazioviana Bail. & Leguminosae \\
\hline 7 & Balfourodendron miedelianum (Engler) Engler & Rutaceae \\
\hline 8 & Bauhinia candicans Benth. & Leguminosae \\
\hline 9 & Blepharocalyx angustifolia Berg & Myrtaceae \\
\hline 10 & Cabralea glaberrima A. Juss. & Meliaceae \\
\hline 11 & Calziandra selzoi (Spreng.) Macbr. & Leguminosae \\
\hline 12 & Campomanesia xanthocarpa (Mart.) Berg & Myrtaceae \\
\hline 13 & Casearia sizvestris Sw. & Flacourtiaceae \\
\hline 14 & Cestrum calycinum willd. & Solanaceae \\
\hline 15 & ChrysophyZZum gonocarpum (Mart. \& Eichl.)Engl. & Sapotaceae \\
\hline 16 & ChrysophyzZum marginatum(Hook. \& Arn.)Radlk. & Sapotaceae \\
\hline 17 & Cupania vernalis Camb. & Sapindaceae \\
\hline 18 & Dalbergia frutescens (Vel1.) Britt. & Leguminosae \\
\hline 19 & Enterolobium contortosiliquum (Vell.) Morong. & Leguminosae \\
\hline 20 & Erythroxylum deciduum St. Hil. & Ery thoxy Zaceae \\
\hline 21 & Eugenia rostrifolia Legr. & Myrtaceae \\
\hline 22 & Eugenia unifiora L. & Myrtaceae \\
\hline 23 & Eugenia sp. & Myrtaceae \\
\hline 24 & Fagara rhoifolia (Lamm.) Engler & Rutaceae \\
\hline 25 & Ficus enormis (Mart. ex Miq.) Miq. & Moraceae \\
\hline 26 & Ficus sp. & Moraceae \\
\hline 27 & Gomidesia selzowiana Berg. & Myrtaceae \\
\hline 28 & Inga marginata Willd. & Leguminosae \\
\hline 29 & Lonchocarpus nitidus (Vog.) Benth. & Leguminosae \\
\hline 30 & Luehea divaricata Mart. & Tiziaceae \\
\hline 31 & Maba inconstans (Jacq.) Griseb. & Ebenaceae \\
\hline 32. & Machaerium stipitatum (DC.) Vog. & Leguminosae \\
\hline 33 & Manihot grahami Hook. & Euphorbiaceae \\
\hline
\end{tabular}


TABELA I. Continuação.

\begin{tabular}{|c|c|c|}
\hline NO & CIENTIFICO & FAMILIA \\
\hline 34 & Matayba elaeagnoides Radlk. & Sapindaceae \\
\hline 35 & Myrceugenia euosma (Berg) Legr. & Myrtaceae \\
\hline 36 & Myrciaria tenelza (DC.) Berg & Myrtaceae \\
\hline 37 & Myrocarpus frondosus FR. Allem. & Leguminosae \\
\hline 38 & Nectandra megapotamica (Spreng.) Nees & Lauraceae \\
\hline 39 & Nectandra saligna Nees ex Mart. & Lauraceae \\
\hline 40 & ocotea puberula Nees & Lauraceae \\
\hline 41 & Parapiptadenia rigida (Benth.) Brenan & Leguminosae \\
\hline 42 & Pouteria gardneriana (A. DC.) Radlk & Sapotaceae \\
\hline 43 & Pouteria salicifolia (Spreng.) Radlk & Sapotaceae \\
\hline 44 & Prunus subcoriacea (Chod. \& Hass1.) Hoehne & Rosaceae \\
\hline 45 & Psychotria cartaginensis Jacq. & Rubiaceae \\
\hline 46 & Randia armata (SW.) DC. & Rubiaceae \\
\hline 47 & Rapanealorentziana Mez & Myrsinaceae \\
\hline 48 & Rapanea umbelzata (Mart.) Mez. & Myrsinaceae \\
\hline 49 & Rollinia exalbida (Vell.) Mart. & Annonaceae \\
\hline 50 & Ruprechtia laxiflora Meissn. & Polygonaceae \\
\hline 51 & Sebastiania klotzschiana Mull. Arg. & Euphorbiaceae \\
\hline 52 & Sebastiania schottiana (Mu11. Arg.)Md11. Arg. & Euphorbiaceae \\
\hline 53 & Solanum verbascifolium L. & Solanaceae \\
\hline 54 & Sorocea bonplandii (Bai11.) Burger & Moraceae \\
\hline 55 & Strychnos brasiliensis (Spreng.) Mart. & Loganiaceae \\
\hline 56 & Tabebuia alba (Cham.) SW. & Bignoniaceae \\
\hline 57 & Tabebuia umbelzata (Sond.) Sandw. & Bignoniaceae \\
\hline 58 & Terminalia australis Camb. & Combretaceae \\
\hline 59 & Trema micrantha(L.) Blume & UZmaceae \\
\hline 60 & Trichilia catigua A. Juss. & Meliaceae \\
\hline 61 & Trichilia elegans A. Juss. & Meliaceae \\
\hline 62 & Trichilia hieronymi Griseb. & Meliaceae \\
\hline 63 & Vitex megapotamica (Spreng.) Mold. & Verbenaceae \\
\hline
\end{tabular}

Desta forma, observa-se na mata ribeirinha uma nitida in fluência da oscilação do nĩvel d'āgua na distribuição das espécies. Sobre o solo pedregoso, resistindo à força da āgua corren te e suportando submersão temporāria, são características: calliandra selloi, Pouteria salicifolia, Sebastiania schottiana e Terminalia austratis.

Em condições mais estāveis ocorrem ainda salix humboldationa, Calliandra tweedii, Celtis spinosa, Ateleia glazioviana, Sebastiania 
klotzchiana, Luehea divaricata, Rapanea ferruginea, Fagara hiemalis, Casearia silvestris, Eugenia uniflora e Aloysia sellowii.

Em zonas mais elevadas a vegetação assemelha-se quanto a

fisionomia e composição florística, à mata secundāria ocorrente na região.

Deve-se ressaltar que, das espécies acima citadas, o tim bó (Ateleia glazioviana) è ārvore típica das regiões fisiográficas do Planalto Mēdio e Alto Uruguai, e que Sebastiania Schottiana e Terminalia australis são espécies abundantes nas matas ciliares da Depressão Central e Campanha.

Na Tabela I, pode-se observar, ainda, que as famílias Le guminosae, Myrtaceae e Euphorbiaceae, são as melhores representadas em nümero de espēcies neste tipo florestal.

Na Tabela I I estão relacionadas as espécies não lenhosas encontradas na mata ribeirinha, predominando os representantes das famīlias Polypodiaceae, Gramineae e Compositae.

TABELA II. NOME CIENTIFICO E FAMILIA BOTÂNICA DAS ESPECIES NAOO LENHOSAS.

\begin{tabular}{|c|c|c|}
\hline Nọ & NOME CIENTIFICO & FAMILIA \\
\hline 1 & Acalypha gracilis spreng. & Euphorbiaceae \\
\hline 2 & Acalypha hispida Burm. & Euphorbiaceae \\
\hline 3 & Acalypha multicaulis Md11. Arg. & Euphorbiaceae \\
\hline 4 & Adiantum cuneatum Langsd. \& Fisch. & Polypodiaceae \\
\hline 5 & Adiantum spp. & Polypodiaceae \\
\hline 6 & Alternanthera micrantha R. E. Fries & Amaranthaceae \\
\hline 7 & Aneimia phyzlitidis (L.) SW. & Schizaeaceae \\
\hline 8 & Aristolochia triangularis Cham. \& Schlecht. & Aristolochiaceae \\
\hline 9 & Arrabidaea chica (H. et B.) Verlot. & Bignoniaceae \\
\hline 10 & Axonopus compressus (SW.) Beauv. & Gramineae \\
\hline 11 & Baccharis sp. & Compositae \\
\hline 12 & Blainvilzea biaristata DC. & Compositae \\
\hline 13 & Blechnum spp. & Polypodiaceae \\
\hline 14 & Bohemeria caudata SW. & Urticaceae \\
\hline 15 & Cardiospermum halicacabulum L. & Sapindaceae \\
\hline 16 & Chaptalia nutans (L.) Hensl. & Compositae \\
\hline 17 & Chome Zia obtusa Cham. \& Schlecht. & Rubiaceae \\
\hline 18 & Chusquea ramosissima Lindm. & Gramineae \\
\hline 19 & Clivia miniata (Hook.) Regel & Amary zzidaceae \\
\hline 20 & Combretum fruticosus (Loefl.) Stuntz & Combretaceae \\
\hline 21 & Cyperus hermafroditus (Jacq.) Standl. & Cyperaceae \\
\hline 22 & Cyperus rotundus $\mathrm{L}$. & Cyperaceae \\
\hline
\end{tabular}


TABELA II. Continuação.

\begin{tabular}{|c|c|c|}
\hline Nọ & NOME CIENTIFICO & FAMILIA \\
\hline 23 & Cуретиs sp. & Cyperaceae \\
\hline 24 & Dalechampia micromeria Baill. & Euphorbiaceae \\
\hline 25 & Desmodium affine Schlecht. & Leguminosae \\
\hline 26 & Desmodium uncinatum (Jacq:) DC. & Leguminosae \\
\hline 27 & Doryopteris multipartita (Feé) Sehnem & Polypodiaceae \\
\hline 28 & Doryopteris sp. & Polypodiaceae \\
\hline 29 & Doxantha ungis-cati (L.) Miers. & Bignoniaceae \\
\hline 30 & Woryopteris spp. & Polypodiaceae \\
\hline 31 & Elephantopus mollis HBR. & Compositae \\
\hline 32 & Hybanthus bigibosus (St. Hil.) Hassl. & Violaceae \\
\hline 33 & Hydrocotyle Zeucocephala Cham. \& Schlecht & Umberziferae \\
\hline 34 & Hypoxis decumbens L. & Amaryzzidaceae \\
\hline 35 & Ichnanthus palzens (Sw.) Munro ex Benth. & Gramineae \\
\hline 36 & Mikania cyananchifolia H. \& A. & Compositae \\
\hline 37 & Nephrolepsis spp. & Polypodiaceae \\
\hline 38 & ocimum sezzoi Berith. & Labiatae \\
\hline 39 & olyra humilis Nees & Gramineae \\
\hline 40 & oplismenus hirtelzus (L.) Beauv. & Gramineae \\
\hline 41 & oplismenus setarius (Lam.) Roem. \& Sch. & Gramineae \\
\hline 42 & oxalis articulata Savigny & oxalidaceae \\
\hline 43 & oxalis mazobolba Cav. & oxalidaceae \\
\hline 44 & Oxalis martiana Zucc. & oxalidaceae \\
\hline 45 & Passiflora elegans Master & Passifzoraceae \\
\hline 46 & Pavonia sepium St: Hil. & MaZvaceae \\
\hline 47 & Petiveria alziacea L. & Phytolaccaceae \\
\hline 48 & Phylzanthus niruri L. & Euphorbiaceae \\
\hline 49 & Piper gaudchaudianum Kunth & Piperaceae \\
\hline 50 & Pithecoctenium achinatum (Jacq.)K. Scham. & Bignoniaceae \\
\hline 51 & Pteridium aquilinum (L.) Ruhn & Polypodiaceae \\
\hline 52 & Relbunium hypocapium (L.) Hemsley & Rubiaceae \\
\hline 53 & Rueliia angustifolia (Nees) Lind. & Acanthaceae \\
\hline 54 & Sarchorachis obtusa (Miq.) Trel. & Piperaceae \\
\hline 55 & Serjania sp. & Sapindaceae \\
\hline 56 & Sida rhombifolia L. & MaZvaceae \\
\hline 57 & Smizax brasiziensis Spreng. & Liliaceae \\
\hline 58 & SmiZax sp. & Liliaceae \\
\hline 59 & Talinum paniculatum (Jacq.) Gaertn. & PortuZaccaceae \\
\hline $\begin{array}{l}60 \\
61 \\
62\end{array}$ & $\begin{array}{l}\text { Tradescantia fluminensis Vell. } \\
\text { Tripogandra elongata (G.G. Meyer) Wood. } \\
\text { Zebrina pendula Schmizl. }\end{array}$ & $\begin{array}{l}\text { Comme linaceae } \\
\text { Comme linaceae } \\
\text { Comme linaceae }\end{array}$ \\
\hline
\end{tabular}


A fisionomia do estrato inferior na mata ribeirinha é,ain da, fortemente caracterizada pela abundāncia de Relbunium hypocar pium, Hydrocotyle leucocephala e espēcies das famīlias oxalidaceae, Piperaceae, Acanthaceae e Cyperaceae. Serjania sp. (Sapindaceae) é a liana mais frequente.

A abundância absoluta e relativa das espécies com $D A P>10 \mathrm{~cm}$ é apresentada na Tabela III. Encontrou-se eelevado número de indivî

TABELA III. ABUNDĀNCIA, DOMINÃNCIA E VALOR DE COBERTURA DAS ESPECIES COM DAP $\geq 10 \mathrm{CM}$.

\begin{tabular}{|c|c|c|c|c|c|c|}
\hline \multirow[b]{2}{*}{ Nọ } & \multirow[b]{2}{*}{ ESPECIES } & \multicolumn{2}{|c|}{ ABUNDANCIA } & \multicolumn{2}{|c|}{ DOMINANCIA } & \multirow{2}{*}{$\begin{array}{l}\text { VALOR DE } \\
\text { COBERTURA }\end{array}$} \\
\hline & & $\begin{array}{l}\text { ABSOLUTA } \\
(\mathrm{NQ} / \mathrm{Ha})\end{array}$ & $\begin{array}{l}\text { RELATIVA } \\
(\%)\end{array}$ & $\begin{array}{r}\text { ABSOLUTA } \\
\left(\mathrm{m}^{2} / \mathrm{Ha}\right)\end{array}$ & $\begin{array}{l}\text { RELATI IVA } \\
(\%)\end{array}$ & \\
\hline 1 & Atahornea triplinervia & 12 & 1,53 & 0,1742 & 0,87 & 1,20 \\
\hline 2 & Allophylus edulis & 8 & 1,02 & 0,1131 & 0,57 & 0,80 \\
\hline 3 & Apuleia Zeiocarpa & 4 & 0,51 & 0,5845 & 2,93 & 1,72 \\
\hline 4 & Atoleia glasioviana & 4 & 0,51 & 0,2020 & 1,01 & 0,76 \\
\hline 5 & Bawhinia candicans & 20 & 2,56 & 0,2730 & 1,37 & 1,96 \\
\hline 6 & Blephorocalyx angustifolius & 4 & 0,51 & 0,0628 & 0,32 & 0,42 \\
\hline 7 & Cabratea glaberrima & 16 & 2,05 & 0,5420 & 2,72 & 2,38 \\
\hline 8 & Campomaneaia xanthocarpa & 28 & 3,58 & 0,9816 & 4,93 & 4,25 \\
\hline 9 & Casearia silveetris & 16 & 2,05 & 0,4165 & 2,09 & 2,07 \\
\hline 10 & Chometia obtusa & 4 & 0,51 & 0,0374 & 0,19 & 0,35 \\
\hline 11 & Chrysophy llum gonocarpum & 36 & 4,60 & 0,8261 & 4,15 & 4,38 \\
\hline 12 & Chrysophy 2lum marginatum & 32 & 4,09 & 0,7825 & 3,93 & 4,01 \\
\hline 13 & Cupania vernalis & 8 & 1.02 & 0,0744 & 0,37 & 0,70 \\
\hline 14 & Enterolobium contortisiliquum & 12 & 1,53 & 0,2375 & 1,19 & 1,36 \\
\hline 15 & Eugenia involucrata & 4 & $0,5 i$ & 0,0374 & 0,19 & 0,35 \\
\hline 16 & Eugenia rostrifolia & 12 & 1,53 & 0,0974 & 0,49 & 1,01 \\
\hline 17 & Eugenia sp. & 4 & 0,51 & 0,0424 & 0,21 & 0,36 \\
\hline 18 & Fiaue organensis & 12 & 1,53 & 0,2077 & 1,04 & 1,29 \\
\hline 19 & Gomidesia sellowiana & 4 & 0,51 & 0,0424 & 0,21 & 0,34 \\
\hline 20 & Inga marginata & 28 & 3,58 & 0,3318 & 1,66 & 2,62 \\
\hline 21 & Lonchocarpus nitidus & 99 & 12,66 & 3,2380 & 16,25 & 14,45 \\
\hline 22 & Luehea divaricata & 12 & 1,53 & 1,6349 & 8,20 & 4,86 \\
\hline 23 & Maba inconstans & 4 & 0,51 & 0,3927 & 1,97 & 1,60 \\
\hline 24 & Machaepium stipitatum & 45 & 5,75 & 0,7096 & 3,56 & 4,65 \\
\hline 25 & Manihot grahami & 4 & 0,51 & 0,4069 & 2,04 & 1,28 \\
\hline 26 & Matayba elaeagnoidea & 44 & 5,63 & 1,3619 & 6,83 & 6,23 \\
\hline 27 & Myrciaria tenella & 4 & 0,51 & 0,0320 & 0,16 & 0,41 \\
\hline 28 & Myrocarpus frondosus & 8 & 1,02 & 0,1742 & 0,87 & 0,95 \\
\hline 29 & Wectandra megapotamica & 61 & 7,80 & 0,6969 & 8,52 & 8,16 \\
\hline 30 & Nectandra saligna & 4 & 0,51 & 0,0602 & 0,30 & 0,36 \\
\hline 31 & Oootea puberula & 16 & 2,05 & 0,2274 & 1,14 & 1,59 \\
\hline 32 & Parapiptadenia migida & 8 & 1,02 & 0,0935 & 0,47 & 0,74 \\
\hline 33 & Pouteria gardneriana & 4 & 0,51 & 0,0656 & 0,33 & 0,44 \\
\hline 34 & Pouteria salioifolia & 40 & 5,12 & 0,8632 & 4,33 & 4,72 \\
\hline 35 & Prunus oubcoriacea & 4 & 0,51 & 0,0395 & 0,20 & 0,36 \\
\hline 36 & Rapanea lorentziana & 28 & 3,58 & 0,8687 & 4,36 & 3,97 \\
\hline 37 & Rapanea umbelzata & 4 & 0,51 & 0,1637 & 0,82 & 0,66 \\
\hline 38 & Rotlinia exalbida & 4 & 0,51 & 0,0493 & 0,25 & 0,38 \\
\hline 39 & Ruprechtia Zaxiflora & 44 & 5,63 & 0,9105 & 4,57 & 5,10 \\
\hline 40 & Sebastiania klotzchiana & 37 & 4,73 & 0,4327 & 2,17 & 3,45 \\
\hline 41 & Tabebuia alba & 4 & 0,51 & 0,0320 & 0,16 & 0,34 \\
\hline 42 & Terminalia australis & 8 & 1.02 & 0,0848 & 0,43 & 0,72 \\
\hline 43 & Trema miorantha & 4 & 0,51 & 0,0493 & 0,24 & 0,38 \\
\hline 44 & Trichilia elegans & 4 & 0,51 & 0,0320 & 0,16 & 0,34 \\
\hline 45 & Vitex megapotamica & 20 & 2,56 & 0,2423 & 1,22 & 1,89 \\
\hline $\begin{array}{ll}T & 0 \\
\end{array}$ & T A L & 778 & 100 & 19,9286 & 100 & 100 \\
\hline
\end{tabular}


duos por hectare (778 ārvores/ha), indicando que a mata ribeirinha na região em estudo encontra-se em estágio inicial de desenvolvimen to. Esta observação é tambēm confirmada pelo grande número de espé cies com ocorrência relativamente homogēnea, das quais salientam-se Lonchocarpus nitidus, Nectandra megapotamica, Machaerium stipitatum, Matayba elaeagnoides, Reprechtia laxiflora, Pouteria salicifolia, Chrysophyllum gonocarpum e Chrysophyllum marginatum.

Da anālise do Valor de Cobertura (Tabela III) pode-se con cluir que as espécies mais características da mata ribeirinha como um todo, são Lonchocarpus nitidus, Nectandra megapotamica, Matayba elaeagnoides, Ruprechtia Iaxiflora, Luehea divaricata, Pouteria sa Zicifolia e Machaerium stipitatum. Deve-se ressaltar, entretanto,que Salixhmboldtiana, Ateleia glazioviana, Terminalia australis e Se bastiania schottiana são também importantes na fisionomia desse tị po florestal especialmente nas proximidades do leito do rio.

A maior parte dos indivíduos encontrados em regeneração natural (Tabela IV) são de espēcies umbrōfilas e abundantes no sub bosque, tais como Actinostemon concolor, Calliandra selloi, Cupania vernalis, Allophylus edulis, Matayba elaeagnoides, Inga marginata e Sebastiania klotzchiana. Com exceção de Calliandra selloi, que è tí pica desse tipo florestal, tratam-se de espécies plásticas e impor tantes na composição fisionômica e estrutural do sub-bosque em ma tas subtropicais do Rio Grande do Sul.

TABELA IV. ABUNDÃNCIA DAS ESPECIES LENHOSAS EM REGENERAÇĀO NATURAL.

\begin{tabular}{llc}
\hline \hline Nọ & \multicolumn{1}{c}{ ESPECIES } & ABUNDANCIA RELATIVA \% \\
\hline 1 & Acacia recurva & 1,09 \\
2 & Actinostemon concolor & 14,78 \\
3 & Allophylus edulis & 5,65 \\
4 & Bauhinia candicans & 2,39 \\
5 & Cabralea glaberrima & 0,22 \\
6 & Calliandra selzoi & 12,83 \\
7 & Campomanesia xanthocarpa & 1,52 \\
8 & Casearia silvestris & 4,13 \\
9 & Cestrum calycinum & 0,43 \\
10 & Chrysophyllum gonocarpum & 1,30 \\
11 & Cupania vernalis & 7,60 \\
12 & Dalbergia frutescens & 0,65 \\
13 & Eugenia rostifolia & 1,74 \\
14 & Eugenia uniflora & 3,26 \\
15 & Inga marginata & 6,30
\end{tabular}


TABELA IV. Continuação.

\begin{tabular}{lll}
\hline \hline Nọ & ESPECIES & ABUNDANCIA RELATIVA \% \\
\hline 16 & Lonchocarpus nitidus & 3,04 \\
17 & Luehea divaricata & 3,26 \\
18 & Machaerium stipitatum & 1,09 \\
19 & Matayba elaeagnoides & 8,70 \\
20 & Myreeugenia euosma & 2,39 \\
21 & Myrciaria tenella & 2,61 \\
22 & Myrocarpus frondosus & 0,22 \\
23 & Nectandra megapotamica & 0,65 \\
24 & Parapiptadenia rigida & 0,87 \\
25 & Prunus subcoriacea & 0,87 \\
26 & Psychotria cartaginensis & 0,22 \\
27 & Randia armata & 0,22 \\
28 & Rapanea lorentziana & 0,65 \\
29 & Rapanea umbellata & 0,43 \\
30 & Rollinia exalbida & 0,65 \\
31 & Sebastiania klotzchiana & 5,43 \\
32 & Solanum verbascifolium & 0,22 \\
33 & Sorocea bonplandii & 2,83 \\
34 & Strychnos brasiliensis & 0,43 \\
35 & Tabebuia umbellata & 0,22 \\
36 & Trichilia catigua & 0,43 \\
37 & Trichilia hieronymi & 0,65 \\
\hline \multirow{2}{*}{ 0 T A L } & $100=51.000$ \\
\hline
\end{tabular}

A regeneração natural das espécies de importância comer cial encontram-se em percentagens significativamente menores. Este fato pode ser atribuído, entre outros fatores, à pequena abundāncia de àrvores matrizes destas espécies.

CONCLUSÕES

A mata ribeirinha apresenta-se em manchas descontínuas na região do curso médio do Rio jacuí, RS, e compõe-se de um número ele vado de espécies arbóreas. Nesta mata, observa-se a ocorrência exclu siva de diversas espécies não presentes em outros tipos da região do referido rio. Do ponto de vista de sua utilização a mata ribeirinha apresenta-se pobre.

ZUSAMMENFASSUNG

LONGHI,S.J.; DURLO,M.A. und MARCHIORI,J.N.C.,1982. Die Ufervegetation der Mittelstrecke des Jacuiflusses, RS. Ciência e Natura(4):151-161. 
Die Zusammensetzung und Struktur des Waldes am Ufer der Mittelstrecke des Jacuiflusses wurde nach verarbeitung von 10 Stick proben characteriziert. Es wurde eine hohe Heterogenitat, grosse Stuckzah1/ha und Anwesenheit einige typische Baumarten getroffen.

\section{REFERENCIAS BIBLIOGRAFICAS}

1. BRAUN-BLANQUET,J. Fitosociologia - Bases para el estudio de las comunidades vegetales. Madrid, H. Blume Ediciones, 1979. 820p.

2. BRUNING,E.F. \& HEUVELDOP,J. Structure and functions in natural and man-made forests in the humild tropics. In: IUFRO WORLD CONGRESS, 16., Norway, 1976. p. 500-511.

3. CAINES,S.A. \& CASTRO,G.M. de O. Application of some phytosologi cal techniques to Brazilian Rain Forest. Amer. J. Bot., 43 (3): 205-217, 1956 .

4. FINOL,U., H. Possibilidades de Manejo Silvicultural para las reservas forestales de la Region occidental. Rev. For.Venez., 12(17): 81-107, 1969 .

5. Nuevos parámetros a considerarse en el anālisis estructu ral de las selvas virgenes tropicales. Rev. For. Venez., 14 (21): 29-42, 1971 .

6 . - Metodos de regeneraciōn natural en algunos tipos de bos ques Venezolanos. Rev. For. Venez., 19(26): 17-44, 1976.

7. FONT-QUER,P. Dicionario de botánica. Barcelona, Labor, 1975. 1244p.

8. FORSTER,M. Strukturanalyses eines tropischen Regenwaldes in Ko lumbien. Alzg. Forst.-u. J.-Ztg., 144 (1): 1-8, 1973.

9. HUECK,K. As florestas da América do Sul. São Paulo, Polígono, 1972. $466 \mathrm{p}$.

10. LAMPRECHT, H. Ensayo sobre unos metodos para el Anālisis Estructu ral de los bosques tropicales. Acta Cientifica Venezolana, 13 (2): $57-65,1962$.

11. Ensayo sobre la estructura florística de la parte sur-orien tal del Bosque Universitario "El Caimital" - Esciado Barinas, Rev. For. Venez., 7(10-11): 77-119, 1964.

12. LONGHI,S.J. A estrutura de uma floresta natura de Araucaria an gustifolia (Bert.) o. Ktze., no sul do Brasil. Curitió, Cur so de Pōs-Graduação em Engenharia Florestą? = UFPr, 1980.198 p. (Dissêrtaçãô ủe Mestrado).

13. PETIT,P.M. Resultados preliminares de unos estudios sobre la re generaciōn natural espontanea en el bosque "El Caimital". Rev. For. Venez., 12(18): 9-21, 1969.

14. RIZZINI, C.T. Nota prévia sobre a divisão fitogeogrāfica do Brasil. Rev. Bras. Geogr., 25(1): 3-64, 1963.

15. RIZZINI,C.T. Tratado de Fitogeografia do Brasil: aspéctos socio 
Lógicos e florísticos. São Paulo, HUCITEC-Ed. da Universida de de São Paulo, 1979. 374 p.

16. VOLKART,C.M. Determinacion de la relacion diametro copa: diame tro tronco en Araucaria angustifolia e Pinus elliottii en la Provincia de Misiones. In: CONGRESO FORESTAL ARGENTINO, 1., Buenos Aires, 1969. Actas del. p. 231-237.

Recebido em agosto, 1982; aceito em outubro, 1982. 
. 